Meta

Journal des tradlucteurs

Translators' Journal

\title{
L'agence de traduction
}

\section{Andrée Quesnel-Bédard}

Volume 21, numéro 1, mars 1976

La traduction et l'entreprise

URI : https://id.erudit.org/iderudit/002014ar

DOI : https://doi.org/10.7202/002014ar

Aller au sommaire du numéro

\section{Éditeur(s)}

Les Presses de l'Université de Montréal

\section{ISSN}

0026-0452 (imprimé)

1492-1421 (numérique)

Découvrir la revue

\section{Citer cet article}

Quesnel-Bédard, A. (1976). L'agence de traduction. Meta, 21(1), 77-80.

https://doi.org/10.7202/002014ar d'utilisation que vous pouvez consulter en ligne.

https://apropos.erudit.org/fr/usagers/politique-dutilisation/ 


\section{L'agence de traduction}

On est tenté de décrire l'agence de traduction en disant ce qu'elle n'est pas et de quels moyens elle est privée. Ce serait plus facile mais le résultat serait une description assez précise d'un service de traduction de l'entreprise publique, ce qui n'est pas mon propos. Par ailleurs, les caractéristiques du travail comme les problèmes varient sans doute d'une agence à l'autre selon sa situation géographique et selon qu'elle est spécialisée ou non. Il convient donc de préciser que la nôttre a pignon sur rue à Ottawa, qu'on pourrait qualifier de microcosme de la nation ou qui, du moins, devrait l'être. De toute manière, le milieu est assez particulier si l'on compte les nombreuses fédérations et associations à caractère national, les conseils, les centrales syndicales qui y ont leur bureau principal ou du moins un représentant: Mentionnons encore trois universités, un océan de ministères et ce qu'il faut d'entreprises de toutes sortes pour répondre aux besoins d'une ville.

Tout le monde ou à peu près a besoin un jour ou l'autre des services d'un traducteur et voilà du même coup l'une des caractéristiques de notre travail : la diversité des connaissances nécessaires, et l'un de ses problèmes les plus agaçants : où trouver, sur-le-champ et sans faute, la documentation et le vocabulaire d'un domaine nouveau ou mal connu? Disons tout de suite qu'il n'est pas toujours possible de refuser, sauf dans le cas où nous n'avons aucun espoir de trouver un expert dans les délais voulus.

Si l'agence s'en tenait à une spécialité, il lui serait relativement facile, en y mettant le prix, de se tenir au courant des nouveautés et de trouver dans les revues spécialisées et à d'autres sources les néologismes aussi nécessaires que hardis qui deviendront monnaie courante après quelques mois d'usage. L'agence qui « fait de tout » ne peut se permettre d'accumuler des tonnes de publications et de retenir les services d'une souris de bibliothèque à seule fin de lui en faire extraire les éléments utiles. D'autre part, l'équipe de traducteurs a rarement le temps, dans le cours d'une journée normale, de réserver une heure ou deux au dépouillement des périodiques, et qui d'entre nous ne souhaite pas de temps à autre une accalmie qui lui permettra de mettre son fichier à jour!

La solution rêvée, à condition encore une fois d'y mettre le prix, serait le terminal relié à une banque de terminologie dotée d'un personnel nombreux, spécialisé, etc. Ce service ne remplacerait évidemment pas le bagage indispensable d'encyclopédies, de dictionnaires, de glossaires de toutes sortes, mais il ajouterait un 
élément de souplesse que tous n'ont pas le temps ou l'occasion de chercher pour leur propre compte. Il y a bien les recueils de mots nouveaux, précieux certes, mais dépassés sitôt parus. Il y a des jeux de fiches dont l'éloge n'est plus à faire mais... il faudrait encore plus. Il faudrait mettre de l'ordre à mesure dans la zone grise où s'accumule chaque jour un fouillis de mots nouveaux dont il reste à préciser le sens et à apprécier l'utilité, qui n'appartiennent proprement à aucun domaine précis et qu'on s'arrache de tous côtés pour les mettre à toutes les sauces. Quant à leur trouver un équivalent, on peut faire appel au milieu : ce n'est pas l'imagination qui y fait défaut. D'ailleurs, ce serait peut-être le moment de mettre en commun les ressources et les talents de l'entreprise publique, des universités et du secteur privé de l'industrie et de la traduction pour alimenter une banque centrale et, en retour d'une contribution raisonnable, mettre à la disposition de tous une remarquable variété de connaissances.

Je songe particulièrement à l'univers de la technique moderne et aux nombreuses disciplines que nous devons toucher assez fréquemment. Là encore, l'agence éprouve des difficultés qui n'existent sans doute pas dans le secteur public. Nous ne pouvons garder sur place plusieurs traducteurs spécialisés, au traitement qui s'impose, pour leur demander deux jours sur trois de faire « un peu de tout ». L'entreprise publique, au contraire, trouve sans peine à utiliser à temps complet les services de traducteurs spécialisés en agronomie, en chimie, en pharmacologie, en relations industrielles peut-être et quoi encore...

Le traducteur employé dans une agence est tout cela à la fois. Qu'on ne me parle pas $\mathrm{du}$ «Jack of all trades, master of none ». Personne ne prétendrait être un expert universel, et j'aimerais bien que l'on reconnaisse une bonne fois la somme de travail que s'impose celui qui doit acquérir dans une foule de domaines des connaissances sinon très étendues, du moins honnêtes. Doit-il nécessairement se condamner à la médiocrité ? Bien sûr, l'actualité, qu'elle soit terminologique ou autre, ne s'administre pas encore en intraveineuses. Tout le monde n'a pas non plus la mémoire encyclopédique ou photographique, mais s'il est un domaine où la déformation professionnelle est précieuse, c'est bien la traduction. Le tout est d'avoir l'œil et l'oreille à l'affût.

Le conseil vaut aussi en matière de recrutement. Comment deviner, au cours d'une entrevue, si le candidat se fera à notre régime ? Certaines natures s'accommodent mieux d'une spécialité qui les tient au chaud comme un vieux veston. Les sources de documentation trouvées, un abonnement à divers périodiques judicieusement choisis et vogue la galère! Votre spécialiste s'installe comme l'huître sur son rocher, aussi longtemps que son domaine est en demande.

J'admets que c'est tentant. D'autre part; le vedettariat n'est pas encore très répandu chez nous et la plupart des traducteurs envisagent une carrière sérieuse, probablement bien rétribuée mais sans grand éclat. Le candidat, fraîchement diplômé peut-être ou étranger à la profession mais qui se sent des aptitudes - c'est plus courant qu'on ne pense - ne doit pas croire pour autant que l'agence n'est qu'une «usine » qui «sort» des mots, des mots et encore des mots, à toute vitesse et sans souci de la qualité. Seulement, il est préférable que l'équipe soit composée de généralistes, quitte à garder en réserve une « écurie » de spécialistes à 
la pige pour les cas particuliers. Nous touchons par la bande, pour ainsi dire, à un autre problème, car l'agence ne dispose pas des mêmes moyens que l'entreprise publique pour recruter son personnel. Un exemple : le gouvernement fédéral peut, à relativement peu de frais, afficher ses demandes de personnel dans les bureaux de poste et les centres de main-d'œuvre du pays entier, tandis que notre périmètre de recherche est beaucoup plus restreint. Ses moyens financiers sont, proportionnellement, plus importants que ceux du secteur privé. Or, dans la capitale nationale où la fonction publique engloutit littéralement les traducteurs et le personnel de soutien, nul ne peut se permettre d'offrir, à exigences égales, des traitements inférieurs aux siens. Le risque serait trop grand d'avoir à remplacer à tout instant ceux que tentent les verts pâturages de Sa Majesté.

Autre problème, corollaire du précédent : la formation coûte relativement plus cher dans le secteur privé. Lorsque le gouvernement envoie ses recrues à l'école des stagiaires, il mobilise pour leur apprendre en quelque sorte les usages de la maison un instructeur qui peut leur donner tous ses soins. Chez nous, un traducteur expérimenté doit se charger d'ouvrir au nouveau venu un monde qui doit lui sembler fait d'un million de particularités, suivant les domaines et les clients. Le traducteur devra aussi, au détriment de son propre rendement, consacrer beaucoup de temps à revoir les travaux du débutant, qui ne restera peut-être que quelques mois ou même, quelques semaines. Toute entreprise court des risques semblables, mais il ne faut pas aussi longtemps dans tous les secteurs pour savoir si le choix a été judicieux. En quelques jours, on peut deviner l'incompétence ou le manque de talent du vendeur, de l'enseignant peut-être, mais en traduction, il est des lacunes qui ne se devinent pas au premier coup d'œil, soit que l'inexpérience les masque temporairement, soit que les premiers travaux n'exigent pas, justement, telles connaissances qui font défaut. Il est probablement plus facile de reconnaitre un choix malheureux dans le traducteur qui n'est pas un débutant, et souvent on mettra fin à l'essai d'un commun accord.

La solution consiste peut-être à mettre au point une épreuve complexe qui ratissera littéralement les connaissances du candidat et permettra d'en dresser un tableau raisonnablement complet, ce qu'on ne peut attendre du traditionnel test de traduction. Ce n'est pas que nous cherchons le phénix; nous n'exigeons même pas de diplôme universitaire en traduction si le candidat présente suffisamment de qualités. Il nous faut toutefois tenir compte des besoins du milieu, et la connaissance de la réalité canadienne est plus précieuse, lorsqu'on traduit un mémoire sur le développement social de telle région, que la plus savante étude sur l'influence du complexe d'Edipe chez Gengis Khan. Qu'on me pardonne ce truisme, mais l'idéal est encore l'équilibre.

Passons vite sur le problème du personnel de soutien qui est sans doute aussi difficile à résoudre dans l'entreprise publique que chez nous. C'est très simple : les secrétaires formées dans de bonnes écoles sont retenues longtemps à l'avance. Or, les bonnes écoles sont loin d'être assez nombreuses. Quant aux jeunes personnes qui sortent d'établissements qui sont plutôt des « usines à dactylos », la formation dont elles ont encore besoin coûte probablement aussi cher à l'employeur qu'elle aurait coûté à l'État si leurs études avaient duré ce qu'il faut. Il y aurait peut-être une solution: créer par des bourses d'études un réservoir de secrétaires formées 
selon les besoins actuels du marché, tout comme le gouvernement fédéral suventionne la formation des traducteurs dont il aura besoin demain et comme certaines grandes maisons préparent la relève de leurs cadres. Le coût ne serait sûrement pas trop élevé en comparaison des avantages, qu'il est inutile d'énumérer.

Un dernier point, particulièrement délicat: la qualité de la traduction. La vigilance ne doit jamais se relâcher, que l'équipe soit composée de débutants ou de vétérans. L'habitude tend d'autres pièges que l'inexpérience mais tout aussi dangereux. Tout cela est bien connu, mais il est un troisième larron qui s'en prend plus volontiers peut-être à l'agence (les pigistes sont priês d'interroger leur mémoire sinon leur conscience). Le client - qui l'eût cru - a aussi ses préférences. Or, les impératifs de la publicité, par exemple, ne cadrent pas nécessairement avec ceux de la syntaxe. Dans trop de domaines, un recueil de directives ou de modes d'emploi risque de dérouter complètement ceux qui n'y trouveront pas le vocabulaire américain familier ou les «équivalents » adoptés au cours des ans et qui ont des « droits d'occupant » fortement enracinés. Les exemples ne manquent pas et je n'ai pas besoin d'insister sur les trésors de diplomatie qu'il faut déployer pour faire abandonner un slogan farci de barbarismes, sans jamais utiliser Grevisse comme argument frappant. Lorsqu'on vous dit : « mettez donc bénéfices marginaux; les employés sont plus habitués à ça », il est bien tentant de rétorquer que si lesdits employés ne sont pas plus bêtes que les autres, ils s'habitueront bien au mot juste.

Ces quelques caractéristiques ne sont sans doute pas celles de toutes les agences de traduction, je l'ai déjà dit. De même, nos problèmes ont peut-être été résolus ailleurs et d'autres agences se heurtent à des difficultés qui ne se présentent pas dans notre milieu. Quant aux solutions qui nous échappent encore, je vois d'ici un «remue-méninges», mise en commun des ressources encore une fois, qui apporterait des réponses adaptées à la diversité des conditions. Dans un pays comme le nôtre, il n'y a jamais de solution universelle. N'écoutez pas ceux qui affirment que nous aurons tout le temps d'y songer lorsque les ordinateurs nous auront mis à la retraite.

ANDRÉE QUESNEL-BÉDARD 